\title{
Clinical and demographic characteristics of childhood neuro-ophthalmology diseases at a tertiary eye care center
}

\author{
Sevim Kuyumcu Kavuncu' ${ }^{1 \oplus}$, Pınar Nalçacıoğlu ${ }^{2 \oplus}$, Gölge Acaroğlu ${ }^{30}$ \\ ${ }^{1}$ Ankara Ulucanlar Eye Training and Research Hospital, Ankara; ${ }^{2}$ Department of Ophthalmology, Ankara Yildırm Beyazıt University \\ Faculty of Medicine, Ankara; ${ }^{3}$ Private Practice, Ankara, Turkey.
}

\begin{abstract}
Background. To evaluate the demographic, etiological, and clinical properties, as well as the treatment modalities of neuro-ophthalmological diseases in childhood.

Methods. We retrospectively analyzed the clinical data of patients younger than 18 years old who were referred to the Neuro-Ophthalmology Department of Ulucanlar Eye Hospital from 2004 to 2019.

Results. Of 1,910 patients who presented to the Neuro-Ophthalmology Department, 128 (6.7\%) were younger than 18 years old at diagnosis, and their data were analyzed. The three most common diagnoses were congenital optic disc (OD) abnormalities in 43 (33.5\%), optic neuropathies in 42 (32.8\%), and idiopathic intracranial hypertension in $11(8.5 \%)$ patients. The most frequent symptoms were as follows: decreased visual acuity in 36 $(28.1 \%)$, headache in $32(25 \%)$, and no symptoms in $19(14.8 \%)$ patients. The best visual prognosis was associated with inflammatory optic neuritis, while hereditary and compressive optic neuropathy resulted in poor visual acuity outcomes.

Conclusions. Congenital OD abnormalities and optic neuropathies are the most frequently seen disorders among children with neuro-ophthalmological diseases. Clinicians should also be aware that children without any symptoms may also have neuro-ophthalmological disorders.
\end{abstract}

Key words: Children, congenital optic disc abnormalities, neuro-ophthalmological diseases, optic neuropathies.

Neuro-ophthalmologic diseases vary from disorders of life-threatening intracranial or systemic diseases to congenital disc anomalies. Moreover, they also have a long-term effect on the visual system and overall development of a child. ${ }^{1}$ Loss of vision at the early stage of life has a negative effect on neurobehavioral development. $^{2}$ Early diagnosis of neuroophthalmological diseases is significant because some of these disorders are treatable and preventable. ${ }^{3}$ Mirdehghan et al. ${ }^{4}$ reported the rate of the prevalence of treatable diseases, such as secondary atrophy of the optic nerve, cortical blindness due to trauma, retinal detachment, and cataract secondary to intrauterine

\footnotetext{
Sevim Kuyumcu Kavuncu
}

sevimkavuncu@hotmail.com

Received 21st June 2020, revised 26th August 2020,

22nd October 2020, accepted 24th December 2020. infections, as $25.7 \%$ in school-aged children. Dhiman et al. ${ }^{5}$ suggested that more than half of optic nerve disease cases could be treatable at an early stage of the disease to prevent visual acuity loss.

There is limited information regarding the various clinical patterns of neuroophthalmological diseases in young ages. It is possible that genetics, race or ethnicity, regional traditions or cultural behaviors, different social and economic conditions, and limited access to medical care centers may result in variability in the diagnosis of neuro-ophthalmological disorders. ${ }^{6-7}$

In this study, we evaluated the causes and clinical characteristics and natural courses of neuro-ophthalmological diseases in children who presented at a neuro-ophthalmology specialty clinic of a tertiary referral eye center in Turkey. 


\section{Material and Methods}

Of the 1910 patients who presented to the Neuro-ophthalmology Department of the Ulucanlar Eye Hospital from 2004 to 2019, the medical records of the $128(6.7 \%)$ patients younger than 18 years old at diagnosis were analyzed retrospectively. The study protocol was approved by the Ethics Committee of Ankara Kecioren Training and Research Hospital. All study procedures were carried out in accordance with the Declaration of Helsinki.

A comprehensive medical history was taken from each participant. Demographic and clinical data were recorded. The mean age of onset of disease, gender, laterality of eye involvement, etiology, complaints at presentation, clinical findings, follow-up period, best-corrected visual acuity (BCVA) with Snellen chart at the initial and final visits, number of visits during the follow-up period, associated systemic diseases and treatment protocols were recorded. All patients' diagnoses and follow-up visits were conducted in the Neuro-Ophthalmology Department by three clinicians (SK, PN, and GA).

During the neuro-ophthalmological examination, we evaluated the color vision using Ishihara's pseudoisochromatic color vision chart. Pupillary light reflexes were checked with a penlight to find any relative afferent pupillary defect (RAPD) and pupillary diameters were measured with the pupil gauge chart in both dim and bright light. Extraocular eye movements were recorded in nine cardinal positions. In addition, anterior segment examination by slit-lamp biomicroscopy and dilated fundus examination with a 90-diopter lens or indirect ophthalmoscopic examination with a 20-diopter lens were performed. If children were unable to read the Snellen E chart, Allen's figures, HOTV, Lea tests or Toddler carts, the visual acuity (VA) was evaluated by counting fingers or from the ability to fix and follow.

Ancillary tests, including a 30-2 or 24-2 visual field test (VFT; Carl Zeiss Humphrey 750
Field Analyzer, Germany), were performed by automatic perimetry. If VFT could not be performed due to patient age and cooperation problems, it was assessed by a confrontation test.

Electrodiagnostic testing (visual evoked potential(VEP)) was performed in patients suspected of optic nerve and visual pathway damage. To make the correct diagnosis, cranial and orbital magnetic resonance imaging (MRI) and cranial MR venography were performed if needed. In addition, if necessary, diagnostic tests like lumbar puncture (LP) were done by a pediatric neurologist.

Especially for diagnosis of congenital optic disc (OD) abnormalities, the patients were examined by B-scan ocular ultrasonography (USG) and when available; optic nerve head autofluorescence imaging by fundus camera or spectral domain optical coherence tomography (SD-OCT). In addition, volumetric OCT scans through the optic nerve head were carried out, and peripapillary retina nerve fiber layer thickness was evaluated by SD-OCT. Anterior segment and fundus photographs were taken when needed.

Optic disc drusen (ODD) was diagnosed as showing refractile calcified hyaline nodules located in the optic nerve head. We accepted significant results in terms of the presented ODD as reported previously. ${ }^{8-10}$ Also, the diagnostic criteria for other congenital optic disc abnormalities (crowded optic disc anomalies, tilted disc syndrome and optic nerve hypoplasia) are as follows the study from Brodsky. ${ }^{11} \mathrm{~A}$ cranial MRI is ordered in all cases with optic nerve hypoplasia to exclude associated intracranial pathologies.

Optic neuritis was determined as acute or subacute unilateral or bilateral visual loss, abnormal color vision, the presence of the RAPD if unilateral or asymmetric involvement, and normal or swollen OD on fundus examination that could not be explained by any other disorder. Bilateral involvement of disease was 
defined as both eyes affected within 1 month, and bilateral recurrent disease was defined as one or both eyes affected more than once. Chronic relapsing inflammatory optic neuritis (CRION) was diagnosed depending on the reported study. ${ }^{12}$

Idiopathic intracranial hypertension (IIH) was determined as elevated intracranial pressure (higher than $\geq 28 \mathrm{~cm} \mathrm{H}_{2} \mathrm{O}$ if the patient was obese or sedated for the process, or $\geq 25 \mathrm{~cm} \mathrm{H}_{2} \mathrm{O}$ if non-obese and non-sedated) for children with normal CSF in combination with normal brain neuroimaging. ${ }^{13}$

Hereditary optic neuropathies were diagnosed with painless, progressive, and permanent bilateral visual loss with central or cecocentral visual field defects and dyschromatopsia after excluding compressive, infiltrative, or infective causes by normal MRI and normal CSF examination if necessary..$^{14}$ Wolfram syndrome is also called DIDMOAD, a term that indicates the components of diabetes insipidus, diabetes mellitus, optic atrophy, and deafness. ${ }^{15}$

Traumatic optic neuropathy was diagnosed as optic nerve dysfunction, including decreased visual loss, RAPD, or dyschromatopsia, following direct or indirect ocular and head trauma that could not be explained by other causes. Furthermore, patients were considered to have acute disseminated encephalomyelitis (ADEM) if they fulfilled the criteria previously reported by the International Pediatric Multiple Sclerosis Study Group. ${ }^{16}$

Compressive or infiltrative optic neuropathy or tumor-related optic neuropathy diagnosis depended on a slow progression of vision loss, the presence of RAPD, abnormal color vision, visual field defect and OD pallor or optic disk edema. The lesions may compress or infiltrate the anterior visual pathway, which is confirmed via cranial and orbital MRI.

Simultaneous changes in pupillary diameter were measured in both bright and dim light to assess anisocoria by using a pupil gauge chart. The diagnosis of pupillary abnormalities, Adie's pupil, and Horner syndrome or physiological anisocoria have been described in a previous study. ${ }^{17}$ Due to the exclusion of the causes of Horner syndrome, we ordered neck and abdominal ultrasonography, cranial and neck MRI, and MRI angiography, as well as enhanced thoracic computed tomography (CT) scanning if needed. ${ }^{18}$

\section{Statistical Analysis}

All statistical analyses were carried out using the SPSS 21.0 statistical analysis program. The descriptive statistics mean or median values, and percentages were obtained in the analysis.

\section{Results}

We identified 128 patients, $6.7 \%$ of our total neuro-ophthalmological patient group, aged less than 18 years. Mean age at onset of disease was $11.9 \pm 3.5$ years (range, $1-18$ years), and median follow-up time was 6 months (range, 3-101 months).

All patients were Turkish Caucasians. There were $71(57.5 \%)$ girls and $57(44.5 \%)$ boys. Among the 128 patients, 83 (64.8\%) presented with bilateral involvement, $43(33.6 \%)$ presented with unilateral involvement, and 2 (1.6\%) did not exhibit ocular involvement. Median number of visits was 3 (1-35). Table I shows the list of diagnoses of neuro-ophthalmological diseases in children and the distribution of gender, ocular involvement, and median age of onset at presentation. We could not find the cause of one case with optic atrophy. This case had no complaint, and optic atrophy was found incidentally on routine eye examination.

Among the compressive etiology of optic neuropathy, the most common presentation was optic nerve glioma $(n=6,66.7 \%)$ associated with neurofibromatosis (NF) type 1 , followed by pituitary adenoma $(n=2,22.2 \%)$ and craniopharyngioma $(n=1,11.1 \%)$. Ocular findings of NF type- 1 cases are shown in Table II. 
Table I. The specific diagnosis of children and distrubition of gender, ocular involvement and the mean age at onset.

\begin{tabular}{|c|c|c|c|c|c|}
\hline Diagnosis & $\begin{array}{l}\text { No. of patients } \\
(\%)\end{array}$ & Gender $(\mathrm{F} / \mathrm{M})(\%)$ & $\begin{array}{l}\text { Unilateral } \\
(\mathrm{n}, \%)\end{array}$ & $\begin{array}{l}\text { Bilateral } \\
(\mathrm{n}, \%)\end{array}$ & $\begin{array}{l}\text { Age at } \\
\text { onset, years }\end{array}$ \\
\hline Congenital OD anom. & $43(33.5 \%)$ & & & & \\
\hline OD drusen & $30(68.9 \%)$ & $17(56.7 \%) / 13(43.3 \%)$ & $7(23.3 \%)$ & $23(76.7 \%)$ & $12.0 \pm 3.0$ \\
\hline Crowded disc & $11(25 \%)$ & $5(45.5 \%) / 6(54.5 \%)$ & $2(18.2 \%)$ & $9(81.8 \%)$ & $9.8 \pm 2.4$ \\
\hline OD Hypoplasia & $1(4.5 \%)$ & $1 \mathrm{~F}$ & -- & $1(100 \%)$ & 13 \\
\hline Tilted disc & $1(2.3 \%)$ & $1 \mathrm{M}$ & -- & $1(100 \%)$ & 14 \\
\hline Optic neuropathy & $42(32.8 \%)$ & & & & \\
\hline Optic neuritis & $14(33.3 \%)$ & & & & \\
\hline Demiyelizan ON & $10(76.9 \%)$ & & & & \\
\hline Isolated ON & $5(38.5 \%)$ & $2(40 \%) / 3(60 \%)$ & $3(60 \%)$ & $2(40 \%)$ & $14(12-18)$ \\
\hline MS-related ON & $3(23.1 \%)$ & $3 \mathrm{~F}$ & $3(100 \%)$ & --- & $14(10-15)$ \\
\hline CRION & $1(7.6 \%)$ & $1 \mathrm{M}$ & --- & $1(100 \%)$ & 15 \\
\hline ADEM & $1(7.6 \%)$ & $1 \mathrm{M}$ & --- & $1(100 \%)$ & 16 \\
\hline Infectious ON & $4(9.5 \%)$ & $1(25 \%) / 3(75 \%)$ & --- & $4(100 \%)$ & $11.5(10-13)$ \\
\hline Herediter ON & $10(23.8 \%)$ & $6(60 \%) / 4(40 \%)$ & --- & $10(100 \%)$ & $11.9 \pm 4.5$ \\
\hline Compressive ON & $9(21.4 \%)$ & $6(66.7 \%) / 3(33.3 \%)$ & $5(55.6 \%)$ & $4(44.4 \%)$ & $11.6 \pm 3.7$ \\
\hline Traumatic ON & $7(16.7 \%)$ & $4(57.1 \%) / 3(42.9 \%)$ & $7(100 \%)$ & --- & $12(10-16)$ \\
\hline Optic atrophy & $1(2.4 \%)$ & $1 \mathrm{M}$ & $1(100 \%)$ & --- & 10 \\
\hline Toxic ON & $1(2.4 \%)$ & $1 \mathrm{M}$ & --- & $1(100 \%)$ & 10 \\
\hline$I I H^{*}$ & $11(8.6 \%)$ & $5(45.5 \%) / 6(54.5 \%)$ & --- & $11(100 \%)$ & $11.7 \pm 3.9$ \\
\hline Pupillary Anomalies & $7(5.5 \%)$ & & & & \\
\hline Horner syndrome & $4(57.1 \%)$ & $1(25 \%) / 3(75 \%)$ & $4(100 \%)$ & --- & $9.5(1-15)$ \\
\hline Adie pupilla & $1(14.3 \%)$ & $1 \mathrm{M}$ & $1(100 \%)$ & --- & 12 \\
\hline Physiologic anisocoria & $1(14.3 \%)$ & $1 \mathrm{M}$ & $1(100 \%)$ & --- & 9 \\
\hline Post-traumatic Mydr. & $1(14.3 \%)$ & $1 \mathrm{M}$ & --- & $1(100 \%)$ & 15 \\
\hline Cranial Nerve Palsies & $6(4.7 \%)$ & & & & \\
\hline $4^{\text {th }}$ nerve palsy & $3(50 \%)$ & $2(66.7 \%) / 1(33.3 \%)$ & $2(66.7 \%)$ & $1(33.3 \%)$ & $10(8-11)$ \\
\hline $3^{\text {th }}$ nerve palsy & $2(33.3 \%)$ & 2 F (100\%) & $2(100 \%)$ & --- & $4.5(2-7)$ \\
\hline $6^{\text {th }}$ nerve palsy & $1(16.7 \%)$ & $1 \mathrm{~F}$ & $1(100 \%)$ & --- & 7 \\
\hline Migraine & $3(2.3 \%)$ & 3 F (100\%) & --- & $3(100 \%)$ & $15(11-15)$ \\
\hline Thyroid Ophthalmopathy & $3(2.3 \%)$ & $3 \mathrm{~F}(100 \%)$ & ---- & $3(100 \%)$ & $16(8-16)$ \\
\hline Secondary IH & $3(2.3 \%)$ & $2(66.7 \%) / 1(33.3 \%)$ & --- & $3(100 \%)$ & $13(10-16)$ \\
\hline Intracranial Tumor & $2(1.6 \%)$ & $2 \mathrm{M}(100 \%)$ & --- & --- & $15(13-17)$ \\
\hline Non-organic VFL & $2(1.6 \%)$ & $2 \mathrm{~F}(100 \%)$ & --- & $2(100 \%)$ & $12(12)$ \\
\hline Other diseases ${ }^{* *}$ & $6(4.7 \%)$ & $5(83.3 \%) / 1(16.7 \%)$ & $4(66.7 \%)$ & $2(33.3 \%)$ & $14(11-17)$ \\
\hline
\end{tabular}

*: Two cases with idiopathic intracranial hypertension also had optic disc drusen in both eye.

**: The number of cases is one for each diagnosis: blepharospasm, nonspesific orbital inflammatory disease, accommodation spasm, oculomotor apraxia, isolated inferior rectus paralysis, carotid-cavernous fistula

$\$$ : mean \pm standard deviation (min-max), median (min-max)

Anom: anomaly, ON: optic neuropathy, CRION: chronic relapsing inflammatory optic neuropathy, ADEM: acute disseminated encephalomyelitis, IIH: idiopathic intracranial hypertension, Mydr: mydriasis, VFL: visual field loss 
Table II. The clinical characteristics of cases with Neurofibromatosis Type-1.

\begin{tabular}{lccc}
\hline Patient & Optic nerve glioma & Cranial glioma & Lisch nodule \\
\hline Case 1 & Unilateral & -- & + \\
Case 2 & Bilateral & -- & + \\
Case 3 & Bilateral & -- & + \\
Case 4 & Bilateral & -- & - \\
Case 5 & -- & + & + \\
Case 6 & -- & + & -- \\
\hline
\end{tabular}

Table III. The clinical features of optic disc drusen.

\begin{tabular}{lll}
\hline Charecteristics & $\mathrm{n}(\%)$ & Age, y, mean \pm SD (min-max) \\
\hline Unilateral/Bilateral & $7(21.9) / 25(78.1)$ & --- \\
Superficial cases & $15(46.9)$ & $11.7 \pm 3.63(6-17) \mathrm{y}$ \\
Buried cases & $9(28.1)$ & $10.5 \pm 3.1(5-14) \mathrm{y}$ \\
Superficial and buried cases & $8(25)$ & $11.5 \pm 1.8(9-14) \mathrm{y}$ \\
\hline
\end{tabular}

n: number of cases, y: years

Table IV. The distribution of causes of ocular motor nerve palsies.

\begin{tabular}{llll}
\hline Etiology & $3^{\text {th }}$ nerve palsy $(\mathrm{n}, \%)$ & $4^{\text {th }}$ nerve palsy $(\mathrm{n}, \%)$ & $6^{\text {th }}$ nerve palsy $(\mathrm{n}, \%)$ \\
\hline Idiopathic & -- & $1(14.3)$ & -- \\
Congenital & -- & $1(14.3)$ & - \\
Trauma & - & -- & $1(14.3)$ \\
Vasculitis & -- & $2(28.6)^{\$}$ & -- \\
Enfection & $1(14.3)^{*}$ & -- & -- \\
Migraine & $1(14.3)^{* *}$ & -- & -- \\
\hline
\end{tabular}

$\mathrm{n}$ : number of eye involvement, ${ }^{\$}:$ The case with left central Horner syndrome , ${ }^{*}$ : Pupillary sparing 3 th nerve palsy, ${ }^{* *}: 3$ th nerve palsy with pupillary involvement

Diagnosis of hereditary optic neuropathy was made with evaluation of clinical findings, and if Leber's hereditary optic neuropathy (LHON) was suspected, genetic testing was ordered. Five cases $(50 \%)$ of our series had a diagnosis of Wolfram syndrome, and five $(50 \%)$ were classified as hereditary optic neuropathy without primary mutations of LHON.

We evaluated 32 cases with OD drusen, with unilateral involvement in 7 (23.3\%), bilateral involvement in $25(78.1 \%)$ cases. Two cases of IIH also had OD drusen in both eyes. The clinical features of OD drusen are shown in Table III. All paralytic cranial nerve cases completely recovered during follow-up period. Causes of ocular motor nerve palsies are summarized in Table IV.
We had four cases with pupillary abnormalities including anisocoria and miosis. All of them had Horner syndrome; one (25\%) congenital case and the other three $(75 \%)$ cases related to migraine. All seven patients with traumatic optic neuropathy were caused by blunt trauma.

Six $(4.7 \%)$ children presented with at least two or three complaints simultaneously at the onset of the disease, and six (4.7\%) children were referred to our clinic by a pediatrician or pediatric neurologist. Table $\mathrm{V}$ summarizes the presenting complaints of children with neuroophthalmologic diseases. Of the patients with IIH, $72.7 \%$ had a headache as the primary complaint. Other symptoms at the onset of disease were blurred vision $(18.1 \%)$ and strabismus (18.1\%). Unlikely, 1 (9\%) of our 
Table V. The presenting complaints at the onset of the neuro-ophthalmological diseases.

\begin{tabular}{ll}
\hline Presenting complaints* & Number of subjects $(\mathrm{n}, \%)$ \\
\hline Vision loss & $36(28.1 \%)$ \\
Headache & $32(25 \%)$ \\
Without compliants & $25(19.5 \%)$ \\
Double vision & $13(10.2 \%)$ \\
Blurry vision & $10(7.8 \%)$ \\
Ptosis & $3(2.3 \%)$ \\
Visual Field loss & $2(1.6 \%)$ \\
Eye lid retraction & $2(1.6 \%)$ \\
Anisocoria & $2(1.6 \%)$ \\
Others* & $3(3.9 \%)$ \\
\hline
\end{tabular}

*: Some subjects had more than one complaint.

**: Others: Proptosis $(n=1,0.8 \%)$, nistagmus $(n=1,0.8 \%)$ and heterocromia $(n=1,0.8 \%)$.

Table VI. The visual acuity distrubition of 213 affected eyes in children with neuro-ophthalmological disorders.

\begin{tabular}{lcc}
\hline \multirow{2}{*}{ Visual acuity } & \multicolumn{2}{c}{ Number of affected eyes $(\mathrm{n} ; \%)$} \\
\cline { 2 - 3 }$\leq 0.1$ & Initial visit & Final visit \\
$0.2-0.5$ & $28(13.1 \%)$ & $20(9.4 \%)$ \\
$\geq 0.6$ & $26(12.2 \%)$ & $24(11.3 \%)$ \\
\hline
\end{tabular}

patients, who was 9 years old, had presented with anisocoria noticed by his parents.

Outcomes of VA of the affected eyes are shown in Table VI. When compared with the results from the initial visit, the final visit showed improvement by at least two lines of Snellen visual acuity in $25(11.7 \%)$ eyes. The VA decreased in $16(7.5 \%)$ and remained unchanged in the other 172 (80.7\%) eyes. The causes of decreased VA were hereditary optic neuropathy in 10 $(66.6 \%)$ eyes, toxic optic neuropathy resulting from Vigabatrin in 2 (13.3\%) eyes, compressive optic neuropathy due to optic nerve glioma in 2 $(13.3 \%)$ eyes, and amblyopia of strabismus with optic nerve drusen in 2 (13.3\%) eyes.

We used ancillary tests to establish a certain diagnosis following the neuroimaging (including cranial and orbital MRI or CT and cranial or MR venography) tests in 92 $(71.9 \%)$ cases, LP in $30(23.4 \%)$ cases, and electrophysiological tests (including VEP) in $12(9.4 \%)$ cases. In our series, we had difficulty in making a correct diagnosis in two cases in which LP was performed with normal pressure. We noticed that we had difficulty differentiating papilledema from anomalous OD in 2 (6.6\%) cases in which LP was performed with normal opening pressure $(<20 \mathrm{~mm} \mathrm{Hg})$ when we retrospectively evaluated 30 patients who underwent LP. One of these was a 10-year-old boy with Down syndrome and another patient was a 10-year-old boy with morbid obesity and blurred vision. Two cases were re-evaluated and diagnosed as crowded OD. The treatment methods employed during the follow-up period are summarized in Table VII.

\section{Discussion}

The present study reports on the demographics, clinical characteristics, and natural courses of neuro-ophthalmological diseases in a population of patients under the age of 18 years. In particular, studies have focused on and describing the etiology of optic atrophy or causes of blindness in children. ${ }^{1,46,19}$ The major cause of ophthalmological examination in $48 \%$ of the 
Table VII. Treatment methods of children with neuro-ophthalmological diseases.

\begin{tabular}{|c|c|c|}
\hline Treatment methods & Diagnosis & Number of cases (n) \\
\hline IV methylprednisolone $\mathrm{a}^{\mathrm{a}}$ & Acute optic neuritis & 10 \\
\hline Oral acetazolamide & $\mathrm{IHH}$ & 8 \\
\hline Oral acetazolamide+ topiramate & $\mathrm{IHH}$ & 3 \\
\hline Radiotherapy+ chemotherapy & NF-1 & 2 \\
\hline Cycloplegic drop & Accommodation spasm & 2 \\
\hline Oral acetazolamide+ IV antibiotics ${ }^{\mathrm{b}}$ & Bacterial meningitis & 1 \\
\hline LP shunt $^{c}$ & $\mathrm{IHH}$ & 1 \\
\hline Tumor excision+ VP shunt & Pinealoma & 1 \\
\hline Tumor excision surgery & Craniopharyngioma & 1 \\
\hline Oral CS+ immunosuppressive agent $\mathrm{t}^{\mathrm{d}}$ & Autoimmune Vasculitis & 1 \\
\hline Total thyroidectomy & Thyroid ophthalmopathy & 1 \\
\hline Covered stent & Carotico-cavernous fistula & 1 \\
\hline
\end{tabular}

a : Treatment protocol for this cases consisted of 10 to $30 \mathrm{mg} / \mathrm{kg} /$ day IV pulse methylprednisolone for 3-5days, followed by oral steroids for 11 days (at a starting dose of $1 \mathrm{mg} / \mathrm{kg}$ )

${ }^{\mathrm{b}}$ : This case resulted in bilateral optic atrophy

c:Symptoms were not controlled by medical treatment and the patient required Lumboperitoneal shunt surgery

$\mathrm{d}: 30 \mathrm{mg} / \mathrm{kg} /$ day oral corticosteroid, and Azathioprine $2.5 \mathrm{mg} / \mathrm{kg} /$ day as immunosuppressive agent

CS: Corticosteroid, IHH: Idiopathic intracranial hypertension, NF-1: Neurofibromatosis type 1, LP shunt: Lumboperitoneal

shunt, VP shunt: Ventriculoperitoneal shunt, IV: İntravenous

children was related to systemic symptoms like seizure, headache, and vomiting. ${ }^{20}$ The others were examined for different kinds of ocular problems, such as strabismus, or underwent routine examination. ${ }^{21}$

In the present study, $28.1 \%$ of the patients applied to our clinic due to visual loss, $25 \%$ for headache, and $19.5 \%$ with no complaints but having abnormalities that were observed in routine eye examination. The most common diagnosis was congenital OD anomalies (33.6\%), and $69.6 \%$ of these patients involved ODD cases in our series. ODD occurs in about $0.4 \%$ of children. ${ }^{20}$ It is often diagnosed incidentally during routine ophthalmological examination, when there are no related symptoms. Some symptoms, such as temporary visual loss. ${ }^{22}$ and visual field loss. ${ }^{23}$ associated with ODD, were reported in studies with data from adult patients rather than children. We observed that the diagnosis of ODD was made incidentally in $54.6 \%$ of cases in our series. However, $45.4 \%$ of patients with ODD applied to our clinic with a headache.
During the childhood period, ODD is usually buried within the optic nerve, but it becomes more superficial as time progresses. Because of this, it is often difficult to diagnose buried drusen properly and differentiate it from papilledema in children. ${ }^{20}$ Hoover et al. ${ }^{24}$ reported that the mean age at which drusen becomes more superficial and visible is 12 years old. The results of our study support this claim; we estimated a mean age of 10.5 years in buried cases and a mean age of 11.7 years in superficial cases.

Some studies have investigated whether the presence of some symptoms and the character of the headache in patients could lead clinicians to perform LP in patients with IIH. The study of the Idiopathic Intracranial Hypertension Treatment Trial Group revealed that headache is the most frequent (84\%) symptom among patients with $\mathrm{IIH} .{ }^{25}$ In contrast, Hamedani et al. ${ }^{26}$ reported that only $10 \%$ of children with IIH had a symptom of headache in their study. We observed that $72.7 \%$ of our patients had a headache as a primary symptom. As in our study, it has been revealed that diagnosing 
ODD depending only on symptoms is not safe because a headache may be present in patients with ODD or IIH. Furthermore, it has been emphasized that the final diagnosis based on a single fundus examination or LP may cause misdiagnosis. ${ }^{27}$ Therefore, using other diagnostic methods, such as OCT, FAF, and B-scan USG, may decrease the possibility of false diagnosis. ${ }^{19,27,28}$ Krishnakumar et al. ${ }^{27}$ examined 15 children with a suspected diagnosis of IIH and reported that only 6 (40\%) out of 15 patients had IIH. In our series, we had difficulty making a correct diagnosis in two cases with crowded disc and performed LP with normal pressure, although both were evaluated and examined by experienced neuro-ophthalmologists with additional OCT and USG findings. Cases with OD abnormality accompanying $\mathrm{IIH}$ are also challenging, although this is rarely seen. In addition, it is more likely to develop papilledema with coexisting risk factors for IIH when ODD or a small cup-to-disc ratio is present. ${ }^{29}$ We had two cases with buried ODD and IIH together.

NF type 1 is one of the most common syndromes coexisting with brain tumors, affecting approximately 1 in 2,500-3,000 people worldwide. $^{30}$ Malignancies of the peripheral nerve sheaths or central nervous system, such as optic nerve glioma or astrocytoma, are often related to NF-1 associated tumors. ${ }^{31}$ Although gliomas frequently settle through the optic pathway (optic nerves, chiasm, tracks, and radiation), they may also arise anywhere in the brain. ${ }^{32}$ Mbekeani et al. ${ }^{6}$ reported that optic glioma was the major reason for children having optic atrophy related to malignancy. Chinta et al. ${ }^{1}$ found that craniopharyngioma (8 patients; $44 \%$ of children with tumors) was the most common cause of compressive optic neuropathy leading to optic atrophy in children. In our series, the most common compressive optic neuropathy was optic nerve glioma due to NF type 1, followed by pituitary adenoma.

Observation of Lisch nodules in the biomicroscopic examination is an important key point for diagnosis. In our series, one of the
NF cases presented with bilateral Lisch nodules and intracranial glioma but no optic pathway glioma involvement; this patient was diagnosed via neuroradiological imaging.

The disease with the best visual prognosis was inflammatory optic neuritis in our cases. A few specific studies have focused on optic neuritis in children; most of the studies have been observational case reports with a limited number of patients. Generally, in the pediatric population, optic neuritis has a good prognosis. Its presentation is usually bilateral, and it occurs after viral infection. In our series, we had cases with mostly bilateral (67\%) rather than unilateral (33\%) involvement, with an equal gender predominance. Optic neuritis is an isolated condition or a component of systemic autoimmune disorders, such as multiple sclerosis (MS), neuromyelitis optica, or ADEM. Some studies have evaluated risk factors in the development of MS. Different studies have reported the rate of development of MS in children, ranging from $4 \%$ to $36 \% \cdot{ }^{33}$ Only three of the cases $(27.3 \%)$ in our series were associated with MS, while others exhibited isolated optic neuritis. This result may depend on the follow-up time of those patients. Increased age, ethnicity, recurring optic neuritis in both eyes or either eye, ${ }^{34,35}$ and the presence of one or more white matter lesions on the brain $\mathrm{MRI}^{36}$ in the first episode of optic neuritis were accepted as related to a higher risk of MS development. All three cases associated with MS were female and had recurrent attacks in both eyes, as well as white matter lesions on the brain MRI at the presentation of optic neuritis. In our series, a high dosage of intravenous methylprednisolone (10-30 $\mathrm{mg} / \mathrm{kg} /$ day) for 3-5 days, followed by oral steroid for 11 days (at a starting dose of $1 \mathrm{mg} / \mathrm{kg}$ ), was given in patients with acute inflammatory optic neuritis; we observed a rapid visual recovery after the treatment. In our series, the final visual acuity of the patients with demyelinating optic neuritis was $\geq 0.6$ in all cases except one which was the case with CRION who had optic atrophy and low visual acuity $<0.1$ 
at the first presentation. A similar observation was also reported by Sun et al., ${ }^{37}$ and this was related to poor visual outcomes and a pale disc at the presentation of the disease. Therefore, it is essential to diagnose diseases early that could present as optic neuritis to prevent permanent visual and neurological dysfunction.

In the present study, most of our cases were involved congenital OD abnormalities and optic neuropathies. The best response to the treatment was in patients with inflammatory optic neuritis. However, the worst prognosis was in cases with hereditary and compressive optic neuropathies. Cases with pupillary abnormalities mostly had migraine, but this symptom may also arise in patients with IIH. It should also be kept in mind that children may exhibit cooperation problems during the examination; moreover, they may not have any complaints even if they have a serious neuroophthalmological disease.

In conclusion, knowledge of the etiology of childhood neuro-ophthalmologic diseases is essential in terms of the clinician's ability to diagnose and treat children with preventable and curable diseases as early as possible. In addition, knowledge of the clinical progression of these disorders will give clinicians the chance to contribute to families' actions to support their children's early rehabilitation and functional improvement.

\section{Author contribution}

The authors confirm contribution to the paper as follows: study conception and design: Sevim Kuyumcu Kavuncu, Pınar Nalçacıoğlu; data collection: Sevim Kuyumcu Kavuncu, Pınar Nalçacıoğlu; analysis and interpretation of results: Sevim Kuyumcu Kavuncu, Pınar Nalçacioğlu; draft manuscript preparation: Sevim Kuyumcu Kavuncu, Pınar Nalçacıoğlu, Gölge Acaroğlu. All authors reviewed the results and approved the final version of the manuscript.

\section{Ethical approval}

The study protocol was approved by the Ethics Committee of Ankara Kecioren Training and Research Hospital. (Number:09.10.2019/1980 )

\section{Source of funding}

No financial support was received for this manuscript.

\section{Conflict of interest}

The authors report no conflicts of interest and have no proprietary interest any of the materials mentioned in this article.

\section{REFERENCES}

1. Chinta S, Wallang BS, Sachdeva V, Gupta A, PatilChhablani P, Kekunnaya R. Etiology and clinical profile of childhood pptic nerve atrophy at a tertiary eye care center in South India. Indian J Ophthalmol 2014; 62: 1003-1007.

2. Kong L, Fry M, Al-Samarraie M, Gilbert C, Steinkuller PG. An update on progress and the changing epidemiology of causes of childhood blindness worldwide. J AAPOS 2012; 16: 501-507.

3. Gilbert CE, Canovas R, Hagan M, Rao S, Foster A. Causes of childhood blindness: results from west Africa, south India and Chile. Eye (Lond) 1993; 7(Pt 1): $184-188$

4. Mirdehghan SA, Dehghan $\mathrm{MH}$, Mohammadpour M, Heidari K, Khosravi M. Causes of severe visual impairment and blindness in schools for visually handicapped children in Iran. Br J Ophthalmol 2005; 89: 612-614.

5. Dhiman R, Singh D, Gantayala SP, Ganesan VL, Sharma P, Saxena R. Neuro-ophthalmology at a tertiary eye care centre in India. J Neuroophthalmol 2018; 38: 308-311.

6. Mbekeani JN, Fattah MA, Poulsen DM, et al. Etiology of optic atrophy: a prospective observational study from Saudi Arabia. Ann Saudi Med 2017; 37: 232239.

7. Lim SA, Wong WL, Fu E, et al. The incidence of neuro-ophthalmic diseases in Singapore: a prospective study in public hospitals. Ophthalmic Epidemiol 2009; 16: 65-73. 
8. Tuğcu B, Özdemir H. Imaging methods in the diagnosis of optic disc drusen. Turk J Ophthalmol 2016; 46: 232-236.

9. Palmer E, Galea J, Crowston JG, Wells AP. Optic nerve head drusen: an update. Neuroophthalmology 2018; 42: 367-384

10. Charg MY, Pineles SL. Optic disc drusen in children. Surv Ophthalmol 2016; 61: 745-758.

11. Brodsky MC. Congenital optic disk anomalies. Surv Ophthalmol 1994; 39: 89-112.

12. Kidd D, Burton B, Plant GT, Graham EM. Chronic relapsing inflammatory optic neuropathy (CRION). Brain 2003; 126(Pt 2): 276-284.

13. Friedman DI, Liu GT, Digre KB. Revised diagnostic criteria for the pseudotumor cerebri syndrome in adults and children. Neurology 2013; 81: 1159-1165.

14. Skidd PM, Lessell S, Cestari DM. Autosomal dominant hereditary optic neuropathy (ADOA): a review of the genetics and clinical manifestations of ADOA and ADOA+. Semin Ophthalmol 2013; 28: 422-426.

15. Urano F. Wolfram syndrome: diagnosis, management, and treatment. Curr Diab Rep 2016; 16: 6.

16. Krupp LB, Tardieu M, Amato MP, et al; International Pediatric Multiple Sclerosis Study Group. International Pediatric Multiple Sclerosis Study Group criteria for pediatric multiple sclerosis and immune-mediated central nervous system demyelinating disorders: revisions to the 2007 definitions. Mult Scler 2013; 19: 1261-1267.

17. Bouffard MA, The pupil. Continuum (Minneap Minn) 2019; 25: 1194-1214.

18. Barrea C, Vigouroux T, Karam J, Milet A, Vaessen S, Misson JP. Horner syndrome in children: a cinical condition with serious underlying disease. Neuropediatrics 2016; 47: 268-272.

19. Mezer E, Chetrit A, Kalter-Leibovici O, Kinori M, Ben-Zion I, Wygnanski-Jaffe T. Trends in the incidence and causes of severe visual impairment and blindness in children from Israel. J AAPOS 2015; 19: 260-265.e1.

20. Chang MY, Pineles SL. Optic disk drusen in children. Surv Ophthalmol 2016; 61: 745-758.

21. Erkkila H. Optic disc drusen in children. Acta Ophthalmol 1977; 55(Suppl 129): 3-44.

22. Sarkies NJ, Sanders MD. Optic disc drusen and episodic visual loss. Br J Ophthalmol 1987; 71: 537539.

23. Davis PL, Jay WM. Optic nerve head drusen. Semin Ophthalmol 2003; 18: 222-242.
24. Hoover DL, Robb RM, Petersen RA. Optic disc drusen in children. J Pediatr Ophthalmol Strabismus 1988; 25: 191-195.

25. Wall M, Kupersmith MJ, Kieburtz KD, et al; NORDIC Idiopathic Intracranial Hypertension Study Group. The idiopathic intracranial hypertension treatment trial: clinical profile at baseline. JAMA Neurol 2014; 71: 693-701.

26. Hamedani AG, Witonsky KFR, Cosico M, et al. Headache characteristics in children with pseudotumor cerebri syndrome, elevated opening pressure without papilledema, and normal opening pressure: a retrospective cohort study. Headache 2018; 58: 1339-1346.

27. Krishnakumar D, Pickard JD, Czosnyka Z, Allen L, Parker A. Idiopathic intracranial hypertension in childhood: pitfalls in diagnosis. Dev Med Child Neurol 2014; 56: 749-755.

28. Gili P, Flores-Rodriguez P, Yanguela J, Herreros Fernandez ML. Using autofluorescence to detect optic nerve head drusen in children. J AAPOS 2013; 17: 568-571.

29. Dai S, Trimboli C, Buncic JR. The optic disc is minimal in children with idiopathic intracranial hypertension. J Child Neurol 2013; 28: 1245-1249.

30. Rasmussen SA, Friedman JM. NF1 gene and neurofibromatosis 1. Am J Epidemiol 2000; 151: 3340.

31. Korf BR. Malignancy in neurofibromatosis type 1. Oncologist 2000; 5: 477-485.

32. Campen CJ, Gutmann DH. Optic pathway gliomas in neurofibromatosis type 1 . J Chid Neurol 2018; 33: 73-81.

33. Pérez-Cambrodí RJ, Gómez-Hurtado Cubillana A, Merino-Suárez ML, Piñero-Llorens DP, LariaOchaita C. Optic neuritis in pediatric population: a review in current tendencies of diagnosis and management. J Optom 2014; 7: 125-130.

34. Waldman AM, Stull LB, Galetta SL, Balcer LJ, Liu GT. Pediatric optic neuritis and risk of multiple sclerosis: meta-analysis of observational studies. J AAPOS 2011; 15: 441-446.

35. Lucchinetti CF, Kiers L, O'Duffy A, et al. Risk factors for developing multiple sclerosis after childhood optic neuritis. Neurology 1997; 49: 1413-1418.

36. Bonhomme GR, Waldman AT, Balcer LJ, et al. Pediatric optic neuritis: brain MRI abnormalities and risk of multiple sclerosis. Neurology 2009; 72: 881-885.

37. Sun MH, Wang HS, Chen KJ, et al. Clinical characteristics of optic neuritis in Taiwanese children. Eye (Lond) 2011; 25: 1457-1464. 